\title{
Experiencia en el tratamiento conservador y quirúrgico en niños con secuelas y complicaciones enfisematosas secundaria a ventilación mecánica: serie de casos y revisión de la literatura
}

\author{
Rogelio Sancho-Hernández,* $\bowtie$ Francisco Javier Cuevas-Schacht,* Gabriel Gutiérrez-Morales, * \\ Adriana Alva-Chaire, ${ }^{*}$ Lizbeth Solorio-Rodríguez ${ }^{*}$
}

*Instituto Nacional de Pediatría, Ciudad de México, México; ${ }^{\dagger}$ Pediatría Integral y Terapia Respiratoria, Toluca, Estado de México.

\begin{abstract}
RESUMEN. Introducción: La enfermedad enfisematosa intersticial es un grupo de afecciones que condicionan atrapamiento ectópico de aire en el intersticio pulmonar del niño en el contexto de un proceso infeccioso y el daño secundario generado por la ventilación mecánica. Se propone una clasificación clínica-radiológica-funcional que permita una orientación diagnóstica y terapéutica. Métodos: Estudio descriptivo, retrospectivo y observacional en una serie de casos con 13 pacientes con los diagnósticos de enfermedad enfisematosa intersticial confirmados por patología y por la evaluación radiológica y funcional. Se dividieron en tres grupos: enfisema lobar intersticial adquirido (ELIA), enfisema intersticial neonatal (EIN) y enfisema intersticial y barotrauma (EIB). Se excluyeron las causas congénitas, se investigaron factores infecciosos y de ventilación mecánica, respuesta a estrategias conservadoras y quirúrgicas, mortalidad y complicaciones. Resultados: En 3 del grupo ELIA se diagnosticaron bronquiolitis obliterante y enfisema intersticial persistente, se practicó resección pulmonar con evolución satisfactoria y en otro biopsia diagnóstica con evolución insidiosa de su vasculopatía hipertensiva; EIN: 4, en 3 la lobectomía erradicó la hipertensión y el cortocircuito pulmonar y se evitó la progresión de la displasia broncopulmonar, y otro respondió al manejo conservador con ventilación de alta frecuencia; en el EIB: 6 recibieron tratamiento con drenaje pleural y representan el grupo de mayor letalidad asociado a neumonía y a presiones ventilatorias elevadas. Conclusiones: Esta clasificación permite una orientación diagnóstica y terapéutica: el grupo ELIA y EIN muestran mejoría pronóstica con la resección pulmonar si las opciones conservadoras han fallado. En el grupo EIB se recomienda el manejo conservador y estrategias ventilatorias suaves.
\end{abstract}

Palabras clave: Enfisema intersticial pulmonar, barotrauma, enfisema lobar intersticial adquirido, enfisema intersticial neonatal, fuga aérea.

Experience in the conservative and surgical treatment in children with sequels and emphysematous complications secondary to mechanical ventilation: series of cases and review of the literature

ABSTRACT. Introduction: Interstitial emphysematous disease (IED) is a group of conditions that affect ectopic air trapping in the pulmonary interstitium of the child in the context of an infectious process and secondary damage generated by mechanical ventilation; a functionalradiological-clinical classification that allows a diagnostic and therapeutic approach is proposed. Methods: A descriptive, retrospective and observational study in cases series with 13 patients with diagnoses confirmed by pathology IED and radiological and functional evaluation. They were divided into 3 groups: interstitial lobar emphysema acquired (ILEA), neonatal interstitial emphysema (NIE) and interstitial emphysema and barotrauma (IEB), congenital causes were excluded, infectious and mechanical ventilation factors, response to conservative and surgical approaches, mortality and complications were investigated. Results: In ILEA group: 3 persistent bronchiolitis obliterans diagnosed interstitial emphysema, pulmonary resection was performed with satisfactory outcome and other diagnostic biopsy with insidious evolution of their hypertensive vascular disease; NIE: 4, 3 lobectomy eradicated hypertension and pulmonary short circuit and progression broncodisplasia avoided, and another responded to conservative management with high frequency ventilation; IEB: 6 were treated with pleural drainage and represent the largest group lethality associated with pneumonia and a high ventilatory pressures. Conclusions: This classification allows

$\triangle$ Autor para correspondencia:

Dr. Rogelio Sancho-Hernández, Instituto Nacional de Pediatría,

Ciudad de México.

Correo electrónico: saherog@yahoo.com.mx

Trabajo recibido: 05-VII-2018; aceptado: 27-XI-2018

Este artículo puede ser consultado en versión completa en http://www.medigraphic.com/neumologia 
a diagnostic and therapeutic orientation: ILEA and NIE group forecasts show improvement with lung resection if conservative options have failed; In the EIB group, conservative management and mild ventilatory strategies are recommended.

Key words: Pulmonary interstitial emphysema, barotrauma, lobar emphysema acquired, neonatal interstitial emphysema, air leak.

\section{INTRODUCCIÓN}

El enfisema pulmonar se caracteriza por una dilatación y destrucción permanente de los espacios aéreos alveolares que se encuentran distales al bronquiolo terminal; por el contrario, el aumento de tamaño de los espacios aéreos alveolares sin datos de destrucción se conoce como hiperinsuflación. El enfisema puede clasificarse según su distribución anatómica dentro del lobulillo pulmonar en cuatro tipos principales: 1) centroacinar; 2) panacinar; 3) paraseptal y 4) irregular; sin embargo, esta clasificación operativa para la población adulta no se aplica a los diversos procesos denominados como hiperinsuflación, enfisema buloso, enfisema intersticial, barotrauma que afectan al pulmón pediátrico y su traducción clínica y funcional resulta imprecisa, originando múltiples términos que conllevan a confusión diagnóstica y terapéutica. El enfisema centroacinar y panacinar del adulto guardan una asociación clara con el tabaquismo y déficit de alfa 1 antitripsina.

En el niño, la asociación con procesos inflamatorios posinfecciosos y el daño secundario generado por la ventilación mecánica explican la mayor parte del enfisema infantil; y sus características histopatológicas de afección intersticial predominante no permite reconocerlas en esta clasificación lobulillar del adulto. El enfisema paraseptal o buloso, el irregular o cicatrizal y otros tipos de enfisema descritos como hiperinsuflación obstructiva y enfisema intersticial, parecen aproximaciones para explicar las descripciones patológicas y funcionales de la afección enfisematosa pulmonar del niño. Se describen a continuación:

a) El enfisema paraseptal: donde la parte proximal del ácino es normal, pero la parte distal es la más afectada, puede extenderse a la vecindad pleural a lo largo de los tabiques del tejido intersticial del lobulillo y aparecer junto a zonas de fibrosis o cicatrización, donde el hallazgo característico son numerosos espacios respiratorios continuos y aumentados de tamaño de apariencia quística, localizados, que miden de $0.5 \mathrm{~mm}$ a más de $2 \mathrm{~cm}$ de diámetro o, inclusive, originar grandes bulas o ampollas subpleurales que al romperse pueden complicar como neumotórax espontáneo secundario. Estas descripciones podrían corresponder con el enfisema buloso y neumotórax espontáneo (EBNE) del adulto. b) El enfisema irregular: afecta irregularmente al ácino y se asocia invariablemente a procesos cicatrizales derivados de procesos inflamatorios, por lo que podría corresponder en la práctica a la forma más frecuente de enfisema infantil. ${ }^{1}$

c) Otras formas de enfisema: corresponden con la hiperinsuflación o enfisema compensador que describe la dilatación de los alvéolos, pero sin destrucción de las paredes septales que se produce en respuesta a la extirpación quirúrgica o funcional de un lóbulo pulmonar, o bien, corresponder a una forma obstructiva en la que el pulmón o el lóbulo afectado se expanden a causa de aire atrapado en su interior por un efecto de válvula donde el agente obstructivo (granuloma, inmadurez o disquinesia del cartílago bronquial) permiten la entrada de aire en inspiración pero no la salida durante la espiración.

A la variante congénita se le ha denominado hiperinflación lobar, y a la originada en el curso de una neumopatía crónica secundario al daño inflamatorio por el volubarotrauma ventilatorio mecánico, la denominaremos enfisema lobar intersticial adquirido (ELIA). ${ }^{2-4}$ Finalmente, otra variante descrita es el enfisema intersticial, que corresponde a una ruptura alveolar con disección ectópica y anormal de aire hacia el estroma del tejido conjuntivo del intersticio pulmonar, que podría migrar al mediastino (neumomediastino), a los canales pleuroperitonales (neumoperitoneo no quirúrgico o neumoretroperitoneo), o al tejido celular subcutáneo (enfisema subcutáneo) donde el barotrauma y el daño inflamatorio, la inmadurez pulmonar, el trastorno parenquimatoso subyacente y el momento fisiopatológico definen para efectos operativos una presentación aguda por enfisema intersticial por barotrauma (EIB) y otra subaguda denominada enfisema intersticial neonatal (EIN). ${ }^{5-7}$

El enfisema intersticial pulmonar (EIP) en los niños, para efectos de este estudio, corresponden a entidades que condicionan un atrapamiento ectópico de aire en el intersticio pulmonar de forma difusa (EIB), localizada (ELIA) o más frecuentemente mixta (EIN) en el contexto de una afección sistémica y pulmonar subyacente. La progresión y la velocidad de acumulación anormal en las estructuras de la unidad respiratoria del niño en crecimiento determinan la afección (obstrucción, restricción, cortocircuito) y explican la 
clínica. La literatura mundial adolece de estadística robusta en torno a estas múltiples afecciones, los reportes aislados resultan anecdóticos y es necesaria una revisión de la literatura.

El propósito de este estudio es presentar una clasificación con un enfoque clínico-radiológicofuncional que permita una orientación diagnóstica en tres grupos y determinar la mejor opción de tratamiento conservador o quirúrgico con base en el pronóstico funcional, la morbilidad y mortalidad en los resultados.

\section{MÉTODOS}

Estudio descriptivo, retrospectivo y observacional en una serie de 13 pacientes durante seis años (febrero 2009 a junio 2016) con los diagnósticos con los que se hace referencia al EIP y confirmados cuando fue posible en las piezas anatómicas posquirúrgicas y con evaluaciones radiológica y funcional respiratorias (gammagrafía pulmonar, el estado clínico crítico de la mayoría de los pacientes y su grupo etario no resultaban apropiados para evaluación de pruebas funcionales respiratorias formales). Se propusieron para su análisis tres grupos: 1) ELIA: donde se incluyeron pacientes en quienes la sobredistensión lobar radiológica y la exclusión funcional lobares se relacionaron con ventilación mecánica prolongada; 2 ) EIN: donde se incluyeron los recién nacidos egresados de la Unidad de Cuidados Intensivos Neonatales con criterios diagnósticos de displasia broncopulmonar (a los 28 días de vida, dependencia de oxígeno y que recibieron alguna modalidad de ventilación mecánica, evidencia radiológica de fuga aérea y de enfisema pulmonar intersticial en sus reportes histopatológicos); y 3 ) EIB: donde se incluyeron los pacientes sometidos a ventilación mecánica por diversas causas, quienes complicaron con síndromes de fuga aérea (neumotórax, neumoperitoneo no quirúrgico, enfisema subcutáneo, neumomediastino). Se excluyeron todas las causas congénitas (hiperinsuflación lobar congénita y otras malformaciones broncopulmonares por el reporte histopatológico), se investigaron los factores de riesgo infecciosos y de asistencia ventilatoria mecánica, así como los resultados respecto a curación, estrategia conservadora y quirúrgicas, morbilidad, mortalidad y complicaciones asociadas.

\section{RESULTADOS}

Se incluyeron 13 pacientes, siendo el sexo masculino el más afectado; en el grupo ELIA se identificaron 3 pacientes en quienes la sobredistensión lobar radiológica y la exclusión funcional lobares se relacionaron con ventilación mecánica prolongada $>30$ días, la hipertensión pulmonar fue la comorbilidad más importante así como la causa de muerte para un paciente que no mostró beneficio con la resección pulmonar del lóbulo enfisematoso afectado y cuyo reporte de biopsia pulmonar mostró vasculopatía arterial hipertrófica; en los otros 2 pacientes se realizó lobectomía superior derecha y en otro neumonectomía, respectivamente, con beneficio en la resolución de la sintomatología y en la extubación temprana en los 3 . En uno de los pacientes sometido a lobectomía el reporte patológico mostró los diagnósticos concomitantes de bronquiolitis obliterante y enfisema intersticial persistente, en el paciente sometido a la neumonectomía por su afección difusa la intervención resultó curativa (figura 1). En el grupo de EIN se identificaron 4 pacientes: 2 de ellos fueron diagnosticados inicialmente como secuelas asociadas a displasia broncopulmonar y los otros 2 como malformaciones congénitas broncopulmonares (hiperinsuflación lobar congénita y malformación
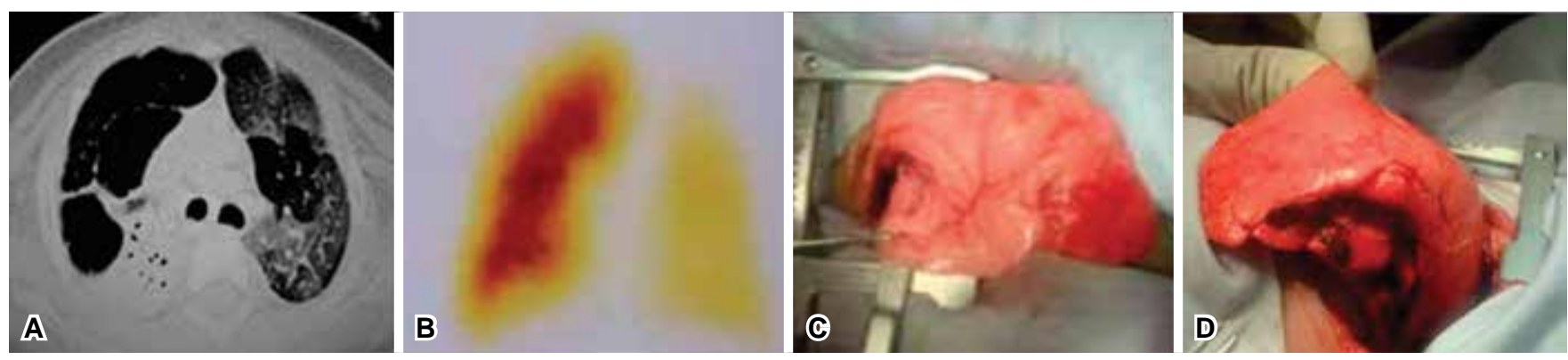

Figura 1. Enfisema lobar intersticial adquirido. A: sobredistensión del lóbulo superior derecho y cambios atelectásicos en lóbulos contiguos por tomografía; B: exclusión funcional del pulmón derecho en vista posterior por gammagrafía; C: aspecto enfisematoso, pálido y nodular del lóbulo superior derecho; D: la lobectomía superior derecha permitió la expansión de los lóbulos medio e inferior previamente atelectásicos y recuperación funcional posterior. 
adenomatoidea quística, respectivamente, ambas etiologías congénitas descartadas por el diagnóstico histopatológico); en 3 de ellos, la resección pulmonar del lóbulo enfisematoso quístico afectado con exclusión funcional resolvieron la sintomatología de cortocircuito pulmonar en todos los casos y es el grupo con mejor pronóstico funcional y clínico y sin mortalidad cuando se relacionaron los casos con los diagnósticos patológicos de enfisema intersticial persistente (3), no encontramos relación directa con los días de ventilación mecánica ni los parámetros ventilatorios descritos en otros reportes (figura 2). El cuarto paciente, al cursar con una sobredistensión hiperlúcida y multiquística, fue catalogado en otro hospital como neumotórax a tensión y conllevó a la inserción errónea de un drenaje pleural que se complicó con síndrome de fuga aérea secundarios, respondió al tratamiento conservador con drenaje pleural, esteroides sistémicos y estrategias ventilatorias de alta frecuencia (VAFO, ventilación de alta frecuencia oscilatoria) con resolución clínica-radiológica de sus lesiones quísticas. No hubo reporte patológico y por su evolución dinámica y hacia la resolución se descartó la posibilidad congénita quística.

En el último grupo de EIB se identificaron 6 pacientes con diagnósticos de neumonía infecciosa comunitaria bilateral (2), de focos múltiples (2) y asociada a ventilador (2), todos relacionados con parámetros ventilatorios elevados $(\mathrm{Pi}>36 \mathrm{mmHg}$ y Peep $>10$ $\mathrm{mm} \mathrm{H}_{2} \mathrm{O}$ ), así como síndrome de dificultad respiratoria aguda (2) y choque séptico (4) quienes evolucionaron con neumotórax, neumomediastino y neumoperitoneo no quirúrgico simultáneamente en los 6 pacientes. En 2 pacientes el neumoperitoneo evolucionó a síndrome compartimental intraabdominal con aumento de la presión intravesical y repercusión hemodinámica, que requirió descompresión con paracentesis y colocación de drenajes intraperitoneales y en un paciente se requirió de un estudio contrastado intestinal para descartar perforación de víscera hueca. En los 6 pacientes se colocaron sellos pleurales con los que remitieron las complicaciones del enfisema subcutáneo por barotrauma, pero 5 de ellos fallecieron por deterioro hemodinámico y gasométrico asociado a la sepsis (4) y otro al SDRA. El único sobreviviente requirió de una traqueostomía por intubación prolongada y ningún paciente fue sometido a técnicas ventilatorias de alta frecuencia (figura 3). En la tabla 1 se describen las características de los pacientes.

\section{DISCUSIÓN}

Con la finalidad académica de contrastar nuestras observaciones con los reportes de caso en la literatura, se describe el análisis en los tres grupos propuestos.

\section{Grupo I: Enfisema lobar intersticial adquirido (ELIA)}

El enfisema lobar adquirido es la expresión «localizada» del enfisema lobar intersticial difuso, por lo cual ELIA parece una descripción más apropiada, algunos autores la designan como «sobredistensión (o hiperinsuflación) lobar enfisematosa»; esta entidad obedece a una ruptura alveolar con infiltración de aire en el intersticio pulmonar perivascular y aunque inicialmente se describió en lactantes con displasia broncopulmonar, también se ha reconocido en síndromes de aspiración meconial, pulmones hipoplásicos, apoyo ventilatorio mecánico con presiones positivas mayores de $30 \mathrm{mmHg}$ en pacientes
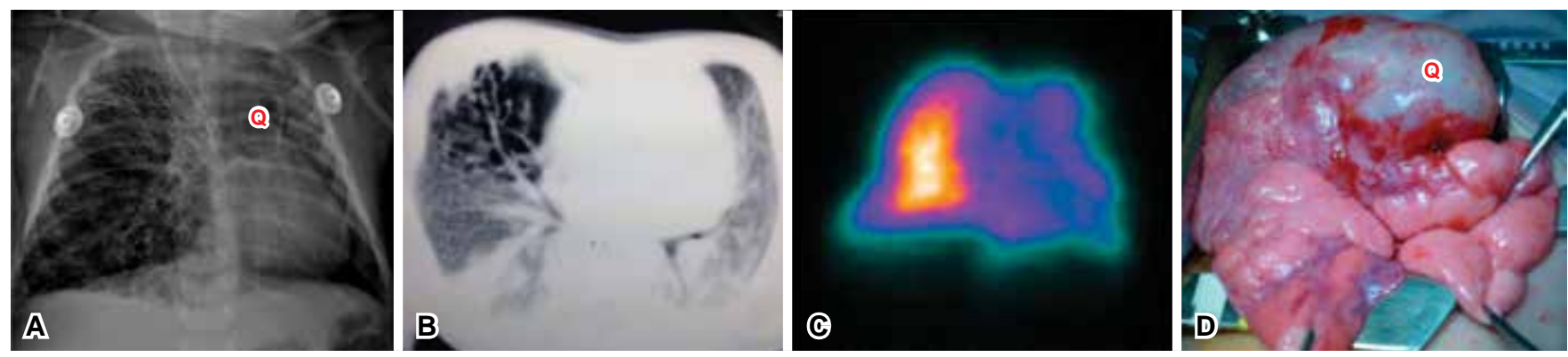

Figura 2. Enfisema intersticial neonatal. A: radiología torácica con sobredistensión lobar y herniación quística del lóbulo superior derecho $(\mathrm{Q})$ hacia el hemitórax contralateral, con cambios quísticos que sugirieron inicialmente malformación adenomatoidea quística tipo II; B: tomografía con cambios reticulonodulares y conglomerados quísticos de predominio en lóbulo superior derecho; C: exclusión funcional del pulmón derecho por gammagrafía en una vista posterior; D: transoperatorio con lóbulo superior enfisematoso, pálido y nodular con degeneración quística (Q) compatible en patología con enfisema intersticial neonatal persistente, la lobectomía permitió la reexpansión anatómica y funcional de los lóbulos residuales. 
neumópatas crónicos y neumonías virales. Numerosos casos cursan con obstrucción bronquial intraluminal por granulomas endobronquiales y estenosis bronquial causados por las complicaciones secundarias de la intubación y trauma endobronquial, o bien, representar obstrucciones dinámicas por colapso de la vía aérea bronquial durante la espiración (broncomalacia) o la disquinesia inflamatoria con traqueoendobronquitis, procesos que deben ser diagnosticados y tratados con broncoscopia.

El análisis anatomopatológico muestra colecciones quístico aéreas en el intersticio perivascular con fibrosis intersticial, disrupción y áreas de atelectasia alveolar, como no existe un estado de disminución del cartílago bronquial se concluye que es una causa adquirida y no congénita. Los efectos del ELIA en el lecho pulmonar, como se describen en nuestros casos expuestos son: disminución de la perfusión del tejido pulmonar afectado, disminución de la ventilación con atrapamiento aéreo, compresión anatómica y funcional del parénquima pulmonar adyacente, tendencia al desarrollo de neumotórax, posibilidad de embolismo aéreo y compresión vascular mediastinal (figura 1). En estos pacientes las indicaciones del tratamiento quirúrgico son:

a) Enfermedad localizada en uno o dos lóbulos pulmonares sin respuesta al tratamiento médico y endoscópico.

b) Deterioro progresivo de la función pulmonar a pesar del apoyo ventilatorio.

c) Sobredistensión de un lóbulo pulmonar involucrado con desviación mediastinal significativa.

d) Compresión de tejido pulmonar adyacente con atelectasia, bronquiectasia y destrucción pulmonar. ${ }^{2-4}$
La lobectomía imprime una mejoría de la función respiratoria, disminución en los requerimientos del apoyo ventilatorio y favorece la extubación, así como la centralización del mediastino en el control radiológico. A pesar de que la lobectomía es inicialmente eficaz para los pacientes seleccionados, debe individualizarse la toma de decisiones durante el transoperatorio para los casos en quienes la lobectomía no ofrece beneficios en la función pulmonar adyacente (imposibilidad de ventilar los lóbulos residuales por atelectasia y fibrosis cicatrizal refractarias) y en cambio agrega factores de morbiletalidad, donde la acción del cirujano, como en uno de nuestros casos, se concreta tan sólo al diagnóstico con una biopsia pulmonar del lóbulo enfisematoso involucrado, puesto que el pronóstico es sugerido por la enfermedad intersticial de origen (figura 4). Las técnicas quirúrgicas reductoras de volumen pulmonar extensamente aceptadas en la población adulta, al complicar con severos síndromes de fuga aérea y fístulas broncopleurales no representan una opción para este enfisema lobar adquirido de la infancia, consideramos que la lobectomía conlleva a un crecimiento compensador del parénquima residual en los menores de 8 años con un pronóstico funcional aceptable y con reducido índice de complicaciones posoperatorias.

\section{Grupo II: Enfisema intersticial neonatal (EIN)}

EI EIN se ha descrito con una incidencia del 33\% en pacientes con bajo peso al nacer y con ventilación mecánica. ${ }^{8,9}$ Los recién nacidos pretérmino están con un riesgo incrementado para EIP debido a un tejido conectivo perivascular más abundante y disecable que en recién nacidos de término, lo que permite que se atrape aire en el espacio intersticial..$^{10,11}$
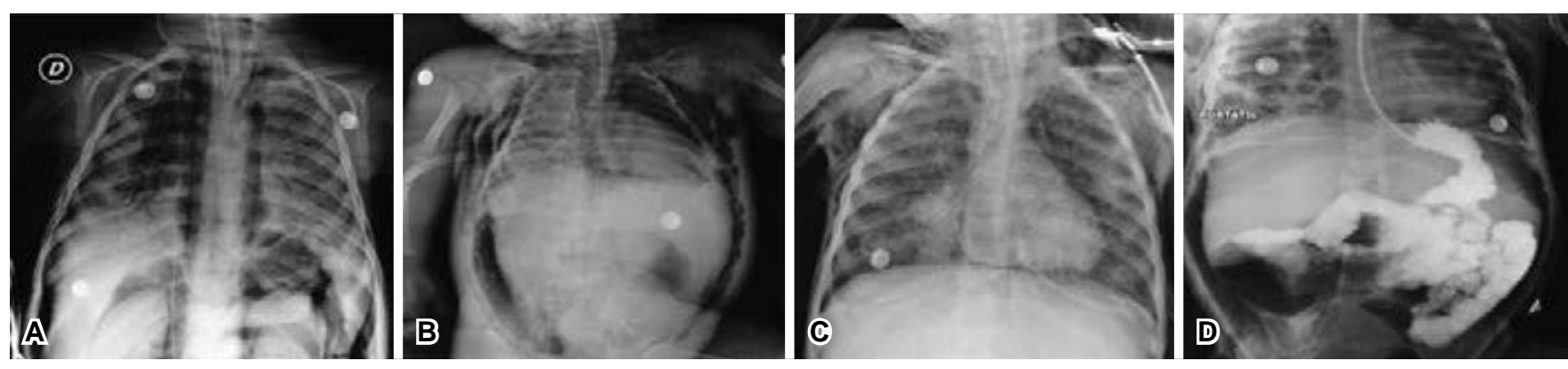

Figura 3. Enfisema intersticial y barotrauma. A: radiología torácica con neumomediastino y neumoretroperitoneo bilateral en el contexto de una neumonía asociada a ventilación mecánica (VM); B: evolución al neumotórax, enfisema subcutáneo y neumoperitoneo asociado a presiones ventilatorias altas; C: remisión gradual posterior a la instauración de sonda pleural conectado a sello de agua y succión negativa; D: estudio contrastado intestinal que confirma la causa del neumoperitoneo no quirúrgico y requirió la colocación de un drenaje intraperitoneal ante datos clínicos de síndrome compartimental intraabdominal. 
Tabla 1. Descripción de los pacientes y análisis de resultados.

\begin{tabular}{|c|c|c|c|}
\hline $\begin{array}{l}\text { Características de } \\
\text { los pacientes }\end{array}$ & $\begin{array}{l}\text { Enfisema lobar intersticial } \\
\text { adquirido (ELIA) } n=3\end{array}$ & $\begin{array}{l}\text { Enfisema intersticial neonatal } \\
\text { (EIN) } n=4\end{array}$ & $\begin{array}{l}\text { Enfisema intersticial por } \\
\text { barotrauma }(\text { EIB) } n=6\end{array}$ \\
\hline Edad/sexo & 3 meses- 1 año/2 M, 1 F & $1-5$ meses $/ 3 \mathrm{M}, 1 \mathrm{~F}$ & 3 meses-13 años $/ 5$ M, 1 F \\
\hline Diagnósticos iniciales & NCI: 2; HLC: 1 & DBP: 2; HLC: 1; MAQ: 1 & NAC 2; NFM 2; NAV 2 \\
\hline $\begin{array}{c}\text { Días con ventilación } \\
\text { mecánica/extubaciones } \\
\text { fallidas }\end{array}$ & $\begin{array}{l}34 \pm 4 \text { días } / 2 \pm 1 \\
\text { extubación fallida }\end{array}$ & $\begin{array}{c}10 \pm 2 \text { días } / 1 \\
\text { extubación fallida }\end{array}$ & $\begin{array}{l}14 \pm 4 \text { días } / 2 \pm 1 \\
\text { extubación fallida }\end{array}$ \\
\hline Parámetros ventilatorios & $\begin{array}{c}\mathrm{Pi} 14 \pm 2 ; \text { Peep } 5 \pm 2 \\
\mathrm{FiO}_{2} 100 \%\end{array}$ & $\begin{array}{l}\mathrm{Pi} 14 \pm 2 ; \text { Peep } 6 \pm 2 ; \mathrm{FiO}_{2} \\
100 \% \text {, ventilación nasal } 2\end{array}$ & $\begin{array}{c}\text { Pi } 36 \pm 4 ; \text { Peep } 13 \pm 2 \\
\mathrm{FiO}_{2} 100 \%\end{array}$ \\
\hline $\begin{array}{l}\text { Agente infeccioso aislado } \\
\text { en secreción bronquial }\end{array}$ & $\begin{array}{c}\text { Adenovirus, } S . \text { aureus, } S . \\
\quad \text { viridans } 1 ; \mathrm{NSI} 2\end{array}$ & C. trachomatis 1 ; NSI: 2 & $\begin{array}{l}\text { P. aeruginosa } 1 ; \text { S. aureus } 1 ; \\
\quad \text { Influenza } A 1 ; \mathrm{NSI} 3\end{array}$ \\
\hline Broncoscopia & Normal 3 & Normal 1; NSI 3 & No se realizó \\
\hline Comorbilidades & Hipertensión pulmonar 2 & NAC con sepsis $1 ;$ NI 1 ; ND 1 & SDRA 2; choque séptico 4 \\
\hline $\begin{array}{c}\text { Tratamiento } \\
\text { conservador y evolución }\end{array}$ & $\begin{array}{c}\text { Intubación selectiva } \\
\text { no tolerada } 1\end{array}$ & $\begin{array}{l}\text { Drenaje pleural 2; } \\
\text { VAFO } 1\end{array}$ & $\begin{array}{l}\text { Drenaje pleural 6; drenaje } \\
\text { intraperitoneal } 2\end{array}$ \\
\hline $\begin{array}{l}\text { Tratamiento } \\
\text { quirúrgico y evolución }\end{array}$ & $\begin{array}{l}\text { LSD 1; biopsia pulmonar } 1 \text {; } \\
\text { neumonectomía derecha } 1\end{array}$ & $\begin{array}{l}\text { Lobectomía superior } \\
\text { izquierda 2; bilobectomía } 1\end{array}$ & $\begin{array}{l}\text { Traqueostomía 1: } \\
\text { único sobreviviente }\end{array}$ \\
\hline $\begin{array}{c}\text { Patología: } \\
\text { diagnósticos finales }\end{array}$ & $\begin{array}{l}\text { ELIA 3: BOC 1; vasculopatía } \\
\text { arterial hipertrófica 1; EIP } 1\end{array}$ & $\begin{array}{l}\text { EIP 3: neumonía por } \\
\text { Citomegalovirus } 1\end{array}$ & No se realizó \\
\hline Mortalidad/causa & $\begin{array}{l}\text { 1/Choque cardiogénico por } \\
\text { hipertensión pulmonar }\end{array}$ & Ninguna & $\begin{array}{l}\text { 5/choque séptico } 4 \\
\text { SDRA } 1\end{array}$ \\
\hline
\end{tabular}

Abreviaturas: $\mathrm{NCI}$ : Neumopatía crónica intersticial; HLC: Hiperinflación lobar congénita; DBP: Displasia broncopulmonar; MAQ: Malformación adenomatoidea quística; BO: Bronquiolitis obliterante; NAC: Neumonía adquirida en comunidad; NFM: Neumonía foco múltiples; NAV: Neumonía asociada ventilador; Pi: Presión inspiratoria; Peep: Presión espiratoria final espiración; NSI: No se identificó; ND: Neumotórax derecho; NI: Neumotórax izquierdo; NB: Neumotórax bilateral; ESC: Enfisema subcutáneo; NP: Neumoperitoneo; SCI: Síndrome compartimental intraabdominal; SDRA: Síndrome de dificultad respiratoria; LSD: Lobectomía superior derecha; BOC: Bronquiolitis obliterante constrictiva; EIP: Enfisema intersticial persistente; VAFO: Ventilación de alta frecuencia oscilatoria.
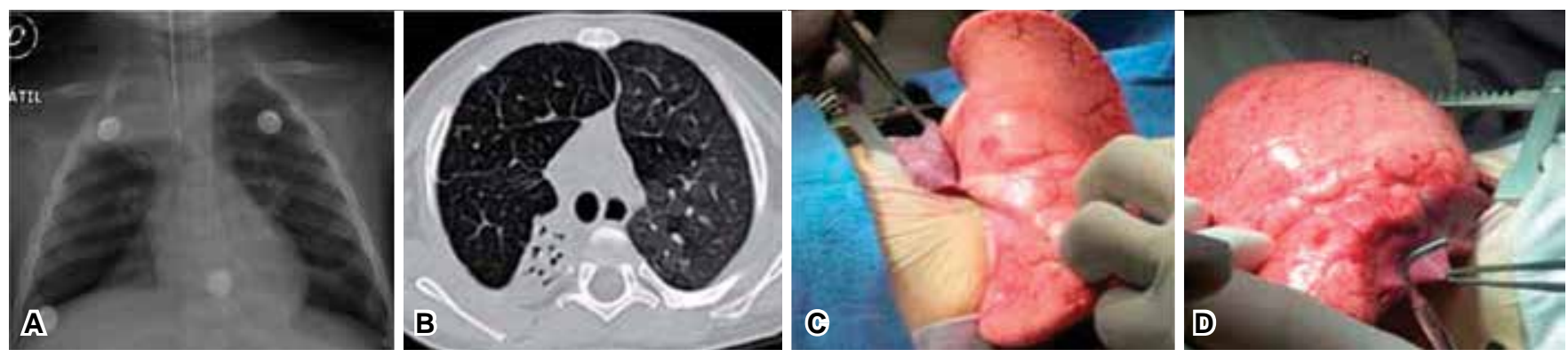

Figura 4. Enfisema lobar intersticial adquirido. A: radiología con atrapamiento aéreo bilateral y atelectasia de los lóbulos superior e inferior derechos en los contornos de una imagen hiperlúcida moderadamente hipertensa; B: en la tomografía gran sobredistensión de los lóbulos superior y medio que condicionan atelectasia del parénquima adyacente y que correspondió con una exclusión total del pulmón derecho por gammagrafía; C: lóbulos superior y medio enfisematosos con cambios atelectásicos y fibróticos irreversibles sobre el lóbulo inferior; D: se decidió tomar dos biopsias pulmonares compatibles por patología con enfisema lobar adquirido y vasculopatía arterial hipertrófica. 
EI EIN ocurre comúnmente como una complicación adquirida y secundaria del barotrauma en recién nacidos con antecedentes de prematurez y consecuente displasia broncopulmonar; obedece en su presentación aguda a sobredistensión con rotura alveolar y fuga de aire dentro del intersticio pulmonar y hacia los canales linfáticos pulmonares evolucionando al neumotórax, neumomediastino, neumopericardio y neumoperitoneo. Típicamente es un fenómeno transitorio pero raramente puede persistir como una conglomeración radiológica quística que puede causar dificultad respiratoria progresiva y ha sido denominado enfisema intersticial pulmonar persistente localizado. ${ }^{12}$ Por guardar un estrecho diagnóstico diferencial con las lesiones congénitas quísticas broncopulmonares la resección quirúrgica es el tratamiento tradicional; sin embargo, existen escasos reportes que remitieron con manejo conservador. ${ }^{13-15}$ Un período entre los datos radiológicos iniciales y el desarrollo de masas quísticas de EIP se describe de 8 a 29 días, promedio de 13 días, información relevante si se aplica al antecedente prácticamente constante de prematurez, ventilación mecánica y cambios radiológicos dinámicos y bilaterales comparado con los hallazgos constantes y estáticos de las lesiones congénitas quísticas broncopulmonares unilaterales. ${ }^{16,17}$

A la evaluación radiológica se corroboran los hallazgos expuestos en nuestros pacientes compatibles con lesiones quísticas radiolúcidas, con sobredistensión pulmonar, múltiples y de paredes delgadas; en las variantes localizadas el lóbulo superior izquierdo es el más afectado, pero en la afección difusa predomina la presentación multilobar y bilateral de los lóbulos inferiores. Podemos considerar a la tomografía como el estudio radiológico de elección donde la apreciación de un patrón heterogéneo de línea-punto (reticulonodular) se ha reportado como específico en el $82 \%$ de los hallazgos tomográficos de esta entidad. Otro beneficio de la tomografía es la capacidad de correlacionar la extensión y los lóbulos involucrados para efectos del diseño quirúrgico. ${ }^{16}$ Se propone un manejo conservador inicial con estrategias ventilatorias de alta frecuencia (VAFO) y maniobras que promueven el decúbito lateral y la intubación selectiva del bronquio contralateral a la EIN con el apoyo diagnóstico y terapéutico de la broncoscopia, así como el estricto control sistémico, antiinflamatorio local, farmacológico y nutricional de la displasia broncopulmonar acompañante..$^{18-22}$ Más del $50 \%$ de los pacientes serán considerados para tratamiento quirúrgico de su afección localizada, las propuestas de indicación quirúrgica para nuestros pacientes son: a) Dificultad respiratoria causada por efecto de masa con desviación mediastinal.

b) Progresión de los síntomas con persistencia o incremento del tamaño de las lesiones.

c) Dificultad para excluir el diagnóstico diferencial con las lesiones quísticas congénitas broncopulmonares, de obligado diagnóstico patológico.

d) Exclusión local y funcional por gammagrafía pulmonar ventilatorio-perfusorio de un lóbulo pulmonar generador de cortocircuito vascular pulmonar (hipertensión pulmonar no cardiogénica) (figura 3). ${ }^{16,23-26}$

Se debe individualizar la decisión entre proporcionar la vigilancia y manejo conservador contra el tratamiento quirúrgico del EIN en aquellos pacientes donde la progresión de la displasia broncopulmonar y el seguimiento mínimo a un año no pueden ser ofertados a los pacientes. En aquellos donde existe una relativa estabilidad clínica y funcional de su afección difusa y bilateral la política expectante y conservadora resulta recomendable.

\section{Grupo III: Enfisema intersticial y barotrauma (EIB)}

El barotrauma es el trastorno producido por un aumento súbito de presión pulmonar que causa enfisema alveolar intersticial, neumotórax, neumomediastino, neumoperitoneo, neumorretroperitoneo y enfisema subcutáneo. ${ }^{27}$ Se produce en zonas pulmonares sometidas a presiones inspiratorias excesivamente elevadas que causan hiperinsuflación y sobredistensión de los alvéolos con rotura alveolar y paso de aire a través del espacio intersticial y perivascular. Los estudios en modelos animales han revelado que las presiones mayores de $40 \mathrm{~cm} \mathrm{H}_{2} \mathrm{O}$ ocasionan enfisema intersticial; las presiones superiores a $50 \mathrm{~cm} \mathrm{H}_{2} \mathrm{O}$ causan enfisema mediastínico y si sobrepasan $60 \mathrm{~cm} \mathrm{H}_{2} \mathrm{O}$ dan lugar a enfisema subcutáneo y neumoperitoneo; estos hechos se pueden extrapolar al paciente pediátrico con factores de riesgo de complicaciones pulmonares como neumonía, síndrome de dificultad respiratoria aguda, prematurez, inmadurez pulmonar, malnutrición, toxicidad por oxígeno, aumento de presión positiva en la vía aérea, enfermedades congénitas y trauma de tórax. Microscópicamente el daño pulmonar consiste en una ruptura del epitelio intersticial con fuga aérea y que puede progresar en sentido distal y causar neumotórax, si el aire viaja por la vainas conjuntivas bronquiales y perivasculares hasta el mediastino, se produce neumomediastino y enfisema subcutáneo; si alcanza el abdomen, a través de los hiatos diafragmáticos causa neumorretroperitoneo y neumoperitoneo no quirúrgico. ${ }^{28}$ 


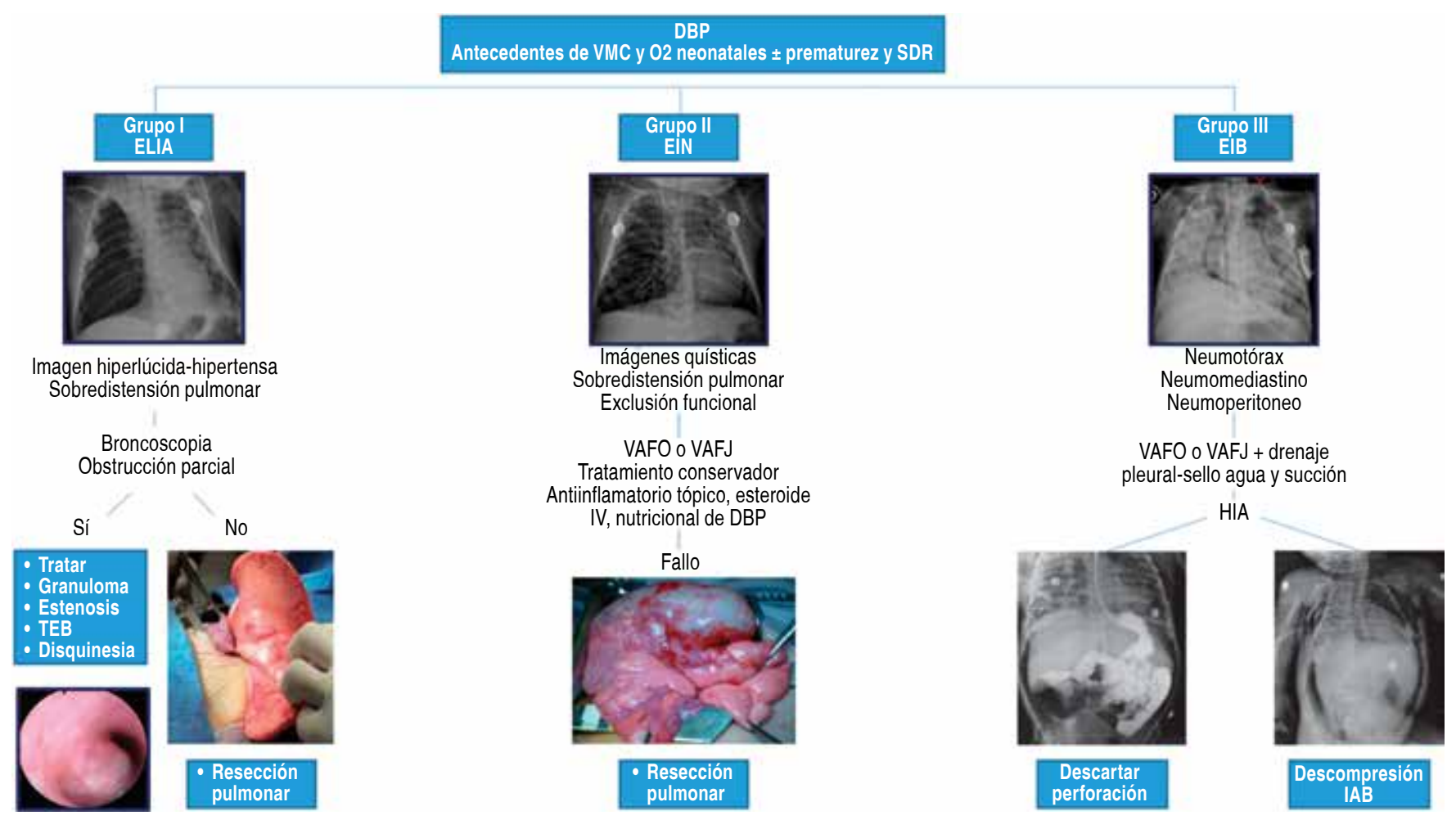

Figura 5. Algoritmo diagnóstico y terapéutico de las secuelas y complicaciones enfisematosas de la ventilación mecánica (VMC) en niños. DBP: displasia broncopulmonar; SDR: síndrome de dificultad respiratoria; ELIA: enfisema lobar intersticial adquirido; EIN: enfisema intersticial neonatal; EIB: enfisema intersticial por barotrauma; VAFO o VAFJ: ventilación de alta frecuencia oscilatoria o jet; HIA: hipertensión intraabdominal; IAB: intraabdominal; TEB: traqueoendobronquitis.

Los estudios contrastados como el tránsito intestinal aportan información valiosa de la integridad intestinal cuando resulta obligado confirmar el diagnóstico diferencial con perforación de víscera hueca dentro del enfoque de un neumoperitoneo no quirúrgico; entre las técnicas quirúrgicas la paracentesis diagnóstica y terapéutica aporta información oportuna de las características del líquido peritoneal (contaminación por contenido gastrointestinal) y mejora las condiciones hemodinámicas del paciente si presenta síndrome abdominal compartimental. El riesgo de neumoperitoneo durante el soporte ventilatorio se debe a una presión inspiratoria máxima (Pim) superior a $40 \mathrm{~cm} \mathrm{H}_{2} \mathrm{O}$ y una presión positiva al final de la espiración (Peep) superior a $6 \mathrm{~cm} \mathrm{H}_{2} \mathrm{O}^{29,30}$ En esta serie de casos las presiones utilizadas fueron Pi de 29 a $40 \mathrm{~cm} \mathrm{H}_{2} \mathrm{O}$ con una Peep de 8 a $18 \mathrm{~cm} \mathrm{H}_{2} \mathrm{O}$, y en todos una $\mathrm{FiO} 2$ del $100 \%$. El tratamiento del barotrauma depende de la repercusión clínica, para el neumotórax con neumomediastino y neumoperitoneo el tratamiento suele ser conservador con estrategias ventilatorias mecánicas encaminadas a disminuir las presiones inspiratorias elevadas y favorecer el reclutamiento alveolar, como son las modalidades de alta frecuencia en sus versiones jet $u$ oscilatorias (VAFO y/o VAFJ), además, de un drenaje pleural con sonda conectada a un sistema de sello de agua y succión negativa; si éste debuta como hipertensión intraabdominal con síndrome compartimental con gran repercusión hemodinámica, ventilatoria y gasométrica es preciso realizar una descompresión abdominal inmediata con paracentesis y colocación de drenaje intraperitoneal (de Penrose, habitualmente uno en cada fosa ilíaca). Aunque se ha descrito la necesidad de una laparotomía descompresiva en ninguno de nuestros pacientes en ausencia de datos de irritación peritoneal fue necesaria y, en lo posible, debe evitarse procedimientos innecesarios que elevan la morbiletalidad.

\section{CONCLUSIÓN}

Esta clasificación permite una orientación diagnóstica y terapéutica pertinente: el grupo ELIA y EIN muestran mejoría funcional y pronóstica con la resección pulmonar en las afecciones localizadas y con fallo a las medidas conservadoras. Finalmente, para el grupo EIB 
se propone manejo no quirúrgico con drenaje pleural y/o abdominal con estrategias ventilatorias gentiles, representa el grupo con mayor mortalidad en asociación con choque séptico y síndrome de dificultad respiratoria. Nuestra casuística es escasa y la revisión de la literatura resulta anecdótica para emitir una recomendación. Esta clasificación se sugiere únicamente para orientar la toma de decisiones en este grupo de pacientes. Se propone un algoritmo diagnóstico y terapéutico de las secuelas y complicaciones enfisematosas de la ventilación mecánica en los niños en la figura 5.

\section{Conflicto de intereses}

Los autores declaran no tener conflicto de intereses.

\section{REFERENCIAS}

1. Kumar V, Abbas AK, Fausto N, Collins J, editores. A Robbins y Cotran A-Patología estructural y funcional. 6a ed. Madrid: McGraw-Hill Interamericana de España; 2000.p.738-742.

2. Martínez-Frontanilla LA, Hernández J, Haase GM, Burrington JD. Surgery of acquired lobar emphysema in the neonate. J Pediatr Surg 1984;19(4):375-379.

3. Azizkhan RG, Grimmer DL, Askin FB, Lacey SR, Merten DF, Wood RE. Acquired lobar emphysema (overinflation): clinical and pathological evaluation of infants requiring lobectomy. J Pediatr Surg 1992;27(8):1145-1152.

4. Sancho-Hernández R, Pérez-Fernández LF, CuevasSchacht FJ. Enfisema lobar adquirido durante una neumopatía crónica intersticial: Implicaciones quirúrgicas y revisión de la literatura. Acta Pediatr Mex 2013;34(4):189-195.

5. Al-Abdi SY, Singhal N. Pulmonary interstitial emphysema and continuous positive airway pressure in a premature infant. Saudi Med J 2005;26(10):1627-1629.

6. Macklin MT, Macklin ChC. Malignant interstitial emphysema of the lungs and mediastinum as an important occult complication in many respiratory diseases and other conditions: an interpretation of the clinical literature in the light of laboratory experiment. Medicine 1944;23(4):281-358.

7. Bas AY, Okumus N, Demirel N, Zenciroglu A. Persistent pulmonary interstitial emphysema in a preterm infant. Indian Pediatr 2008;45(9):775-777.

8. Greenough A, Dixon AK, Roberton NR. Pulmonary interstitial emphysema. Arch Dis Child 1984;59(11):10461051.

9. Crosswell HE, Stewart DL. Special feature: radiological case of the month. Pulmonary interstitial emphysema in a nonventilated preterm infant. Arch Pediatr Adolesc Med 2001;155(5):615-616.

10. Gronbach J, Ehrhardt H, Zimmer KP, Waitz M. Early pulmonary interstitial emphysema in preterm neonates-respiratory management and case report in nonventilated very low birth weight twins. AJP Rep 2018;8(2):e99-e105. doi: 10.1055/s-0038-1648253.

11. Wiswell TE, Srinivasan P. Continuous positive airway pressure. In: Goldsmith JP, Karotkin EH, editors. Assisted ventilation of the neonate. 4th ed. Philadelphia, USA: Saunders; 2003.p.127-147.

12. Kemper AC, Steinberg KP, Stern EJ. Pulmonary interstitial emphysema: CT findings. AJR Am J Roentgenol 1999;172(6):1642.

13. Fitzgerald D, Willis D, Usher R, Outerbridge E, Davis GM. Dexamethasone for pulmonary interstitial emphysema in preterm infants. Biol Neonate 1998;73(1):34-39.

14. Squires KA, De Paoli AG, Williams C, Dargaville PA. High-frequency oscillatory ventilation with low oscillatory frequency in pulmonary interstitial emphysema. Neonatology 2013;104(4):243-249. doi: 10.1159/000353376.

15. Jassal MS, Benson JE, Mogayzel PJ Jr. Spontaneous resolution of diffuse persistent pulmonary interstitial emphysema. Pediatr Pulmonol 2008;43(6):615-619. doi: 10.1002/ppul.20820.

16. Jabra AA, Fishman EK, Shehata BM, Perlman EJ. Localized persistent pulmonary interstitial emphysema: CT findings with radiographic-pathologic correlation. AJR Am J Roentgenol 1997;169(5):1381-1384.

17. Donnelly LF, Lucaya J, Ozelame V, et al. CT findings and temporal course of persistent pulmonary interstitial emphysema in neonates: a multiinstitutional study. AJR Am J Roentgenol 2003;180(4):1129-1133.

18. Keszler M, Donn SM, Bucciarelli RL, et al. Multicenter controlled trial comparing high-frequency jet ventilation and conventional mechanical ventilation in newborn infants with pulmonary interstitial emphysema. J Pediatr 1991;119(1 Pt 1):85-93.

19. Mahapatra S, Scottoline B. Steroid-induced resolution of refractory pulmonary interstitial emphysema. J Matern Fetal Neonatal Med 2016;29(24):4092-4095. doi: 10.3109/14767058.2016.1159673.

20. Mohsini K, Reid D, Tanswell K. Resolution of acquired lobar emphysema with dexamethasone therapy. J Pediatr 1987;111(6 Pt 1):901-904.

21. Dickman GL, Short BL, Krauss DR. Selective bronchial intubation in the management of unilateral pulmonary interstitial emphysema. Am J Dis Child 1977;131(3):365.

22. Wood RE. Clinical applications of ultrathin flexible bronchoscopes. Pediatr Pulmonol 1985;1(5):244-248.

23. Stocker JT, Madewell JE. Persistent interstitial pulmonary emphysema: another complication of the respiratory distress syndrome. Pediatrics 1977;59(6):847-857.

24. Belcher E, Abbasi MA, Hansell DM, Ffolkes L, Nicholson AG, Goldstraw P. Persistent interstitial pulmonary emphysema requiring pneumonectomy. J Thorac Cardiovasc Surg 2009;138(1):237-239. doi: 10.1016/j.jtcvs.2008.06.013.

25. Carey B. Neonatal air leaks: pneumothorax, pneumomediastinum, pulmonary interstitial emphysema, pneumopericardium. Neonatal Netw 1999;18(8):81-84.

26. Leonidas JC, Moylan FM, Kahn PC, Ramenofsky ML. Ventilation-perfusion scans in neonatal regional 
pulmonary emphysema complicating ventilator assistance. AJR Am J Roentgenol 1978;131(2):243-246.

27. Shapiro JM. Intensive care management of status asthmaticus. Chest 2001;120(5):1439-1441.

28. Grosfeld JL, Boger D, Clatworthy HW Jr. Hemodynamic and manometric observations in experimental air-block syndrome. J Pediatr Surg 1971;6(3):339-344.
29. Cruz-Ramos J, Pérez-Fernández LF, Herrera-García W, Granados-Navas FJ. Neumoperitoneo causado por ventilación mecánica asistida. Acta Pediatr Mex 2010;31(4):168-173.

30. Altman AR, Johnson TH. Pneumoperitoneum and pneumoretroperitoneum. Consequences of positive endexpiratory pressure therapy. Arch Surg 1979;114(2):208-211. 